\section{Lung Ultrasound in COVID-19}

Chest $X$-ray is considered less sensitive in diagnosing coronavirus disease 2019 (COVID-19) pneumonia, and due to the milder disease and relatively subtle findings [1], the sensitivity may be further lowered in children. Though chest computerized tomography (CT) is the imaging modality of choice in COVID-19, difficulties in sparing a dedicated machine, transferring potentially infectious and sick patients to the $\mathrm{CT}$ room, disinfection of machine, and ionizing radiation exposure make it less appealing, especially in children. Therefore, a readily available, point-of-care tool that avoids radiation exposure is needed.

Lung ultrasound (LUS) is routinely used as a point-of-care imaging tool in emergency and intensive care units, and its role in COVID-19 is being explored. COVID-19 classically presents as diffuse bilateral pneumonia with asymmetric patchy lesions in the lung periphery that are amenable to ultrasound visualization [2]. Its easy availability, easy decontamination, freedom from radiation, and portability favor its use in COVID-19.

All 14 lung areas (three posterior, two lateral, and two anterior) should be scanned. B-lines are the most classical findings of COVID-19 pneumonia and some authors describe COVID-19 pneumonia as a "storm of clusters of B-lines", sometimes appearing as shining white lung [2]. In a metaanalysis of seven studies (122 patients), almost all patients had abnormal LUS [3]. The common abnormalities were interstitial involvement/B-pattern $(97 \%)$, pleural line abnormalities (70\%), pleural thickening (54\%), consolidation $(39 \%)$, and pleural effusion (14\%) [3]. The number and appearance of B-lines also correlate with the disease severity. As disease progresses, the B-lines increase in number and become more confluent. In severe disease, extensive areas of subpleural consolidations and pleural effusion may be visualized. On serial monitoring, a decrease in the B-lines and appearance of A-lines indicate recovery [4].
Till now only three studies ( 23 patients) have evaluated the role of LUS in pediatric patients. Most common findings were pulmonary interstitial syndrome (82\%) followed by consolidation. Only one study (5 patients) directly compared chest CT, $X$-ray, and LUS and found that ultrasound fares better than chest $X$-ray [5].

Few important limitations of LUS are the inability to detect deep and intrapulmonary lesions, difficult to scan posterobasal regions in sick patients, and relatively lower sensitivity than CT scan. Point of care lung ultrasound, where available, may be utilized in the management of children with COVID-19.

Published online: July 24, 2020; PII: S097475591600215

ARUSHI YADAV ${ }^{1}$ AND JOGENDER KUMAR ${ }^{2}$ *

${ }^{1}$ Department of Radiodiagnosis, Government Medical College and Hospital; and ${ }^{2}$ Department of Pediatrics, Post Graduate Institute of Medical Education and Research; Chandigah, India.

\section{REFERENCES}

*jogendrayadv@gmail.com

1. Balasubramanian S, Rao NM, Goenka A, Roderick M, Ramanan AV. Coronavirus disease 2019 (COVID-19) in children - What we know so far and what we do not. Indian Pediatr. 2020;57:435-42.

2. Volpicelli G, Gargani L. Sonographic signs and patterns of COVID-19 pneumonia. Ultrasound J. 2020;12:22.

3. Mohamed MFH, Al-Shokri S, Yousaf Z, Danjuma M, Parambil J, Mohamed S, et al. Frequency of abnormalities detected by point-of-care lung ultrasound in symptomatic COVID-19 patients: Systematic review and meta-analysis. Am J Trop Med Hyg. 2020. Available from: http:// www.ajtmh.org/content/journals/10.4269/ajtmh.20-0371. Accessed June 17, 2020.

4. Fiala MJ. Ultrasound in COVID-19: A timeline of ultrasound findings in relation to CT. Clin Radiol. 2020;75:553-4.

5. Feng XY, Tao XW, Zeng LK, Wang WQ, Li G. Application of pulmonary ultrasound in the diagnosis of COVID-19 pneumonia in neonates. Zhonghua $\mathrm{Er} \mathrm{Ke} \mathrm{Za} \mathrm{Zhi.}$ 2020;58:347-50.

\section{Pubertal Menorrhagia-A Rare Presentation of Congenital Factor XIII Deficiency}

Factor XIII deficiency, with an estimated incidence of 1 in 1-2 million, is an extremely rare congenital bleeding disorder with autosomal recessive inheritance and often with a history of consanguinity [1]. It can present with myriad bleeding manifestations in different age groups, with varying severity. This is partly due to multiple possible mutations of the two subunits of Factor XIII (subunit A mutations are associated with severe manifestations than those of subunit B) and partly because factor XIII is a multifunctional protein (required in wound healing, maintenance of pregnancy, angiogenesis and hemostasis). Umbilical cord bleeding, present in $73 \%$ of patients, is highly suggestive of this condition [1,2]. Pubertal menorrhagia was present in nearly $31 \%$ patients as per an Indian study [1].

A14-year-old girl presented with severe anemia and 
menorrhagia, refractory to antifibrinolytic therapy, during the third menstrual cycle, with significant past history of having menorrhagia in every cycle after the onset of menarche. These episodes were being treated with blood transfusions, antifibrinolytics, hematinics and oral contraceptive pills. She was born as a third issue of third degree consanguineous couple with no relevant family history and bleeding diathesis. Ultrasound of pelvis, thyroid profile, platelet counts and morphology, coagulation profile (PT-12.1sec, PTT-28sec, INR-1) and clot retraction tests were normal. With this background, qualitative assay for factor XIII was considered and clot solubility test was positive. Quantitative assay, platelet function test, von Will ebrand factor assay and molecular genetic test were not performed because of affordability issues.

Although, coagulopathy is the second most common cause of pubertal menorrhagia [3] with congenital factor XIII deficiency being the commonest amongst the 'rare congenital factor deficiencies', it stays under diagnosed due to its rarity, heterogeneity and absence of diagnostic facilities [1]. Qualitative assay with clot solubility in 5M urea detects only severe form, but quantitative functional assay detects all forms and it is the recommended first line screening method as it has no false positivity [4]. The available treatment options include replacement and prophylactic therapy with cryoprecipitate, fresh frozen plasma and factor XIII concentrates [5].

Awareness about all possible features of factor XIII deficiency is essential among clinicians while managing cases for prompt diagnosis, appropriate treatment, improving quality of life, decreasing the burden of further medical catastrophes, follow up and genetic counseling to prevent morbidity in affected children.

Acknowledgments: Dr Spoorthi SM and Dr Lingaraja Gowda C Patil for help in data analysis and drafting the manuscript.

SN NIKITHA RAJ* AND H RAMESH
Department of Pediatrics,
JJMMedical College, Davanagere,
Karnataka, India.
*snnikitharaj@gmail.com

\section{REFERRENCES}

1. Shetty S, Shelar T, Mirgal D, Nawadkar V, Pinto S, Shabhag $\mathrm{S}$, et al. Rare coagulation factor deficiencies: A countrywide screening data from India. Haemophilia. 2014;20:575-81.

2. Naderi M, Dorgalaleh A, Alizadeh S, Taibibian S, Hosseini $\mathrm{S}$, Shamsizadeh $\mathrm{M}$, et al. Clinical manifestations and management of life threatening bleeding in the largest group of patients with severe factor XIII deficiency. Int $\mathrm{J}$ Hematol. 2014;100:443-9.

3. Karaman K, Ceylan N, Karaman E, Akbayram S, Akbaryram HT, Garipardic M, et al. Evaluation of the hemostatic disorders in adolescent girls with menorrhagia: Experiences from a tertiary referral hospital. Indian $\mathrm{J}$ Hematol Blood Transfus. 2016;32:356-61.

4. Kohler HP, Ichinose A, Seitz R, Ariens RAS, Muszbek L. Diagnosis and classification of factor XIII deficiencies. J Thromb Haemost. 2011;9:1404-06.

5. Jennings I, Kitchen S, Woods TAL, Preston FE. Problems relatedto the laboratory diagnosis of factor XIII deficiency: A UK NEQAS study. J Thromb Haemostat. 2003;1:2603-8.

\section{Arrhythmias Associated with Administration of Anti-fungal Agents}

An 8-year-old boy who had received a bone marrow transplant due to relapse of acute lymphoblastic leukemia was admitted for veno-occlusive disease. He also developed acute kidney injury and was dialysis dependent. His condition was gradually stabilized but he subsequently developed invasive pulmonary aspergillosis with multiple aspergillomas involving both lungs. He was then given oral posaconazole $300 \mathrm{mg}$ twice daily and intravenous liposomal amphotericin B (Ambisome) $90 \mathrm{mg}(3 \mathrm{mg} / \mathrm{kg})$ daily infused through a Hickman catheter over one hour. The tip of the central catheter was located at the junction between superior vena cava (SVC) and right atrium (RA). However, he developed feeding intolerance with severe abdominal pain requiring temporary suspension of enteral feeding. Posaconazole was switched to intravenous voriconazole $210 \mathrm{mg}$ every 12 hours. Five days after the coadministration of voriconazole and amphotericin B infusion, he developed an attack of non-sustained wide complex tachycardia lasting for 24 seconds after infusion of voriconazole and amphotericin B. Thirty seconds later, there were three similar attacks lasting for 15,2 and 4 seconds, respectively with an interval duration of 1 second in between. He was asymptomatic during the attacks. He was receiving continuous renal replacement therapy at that juncture. An electrocardiogram performed immediately after the event failed to capture the ventricular tachycardia. It showed a QT interval of 0.42 seconds. Few days later, the asymptomatic ventricular ectopics appeared again during amphotericin B infusion. There were no other pro-arrhythmic medications, and the tacrolimus level was $5.3 \mu \mathrm{g} / \mathrm{L}$. Voriconazole was then switched back to oral posaconazole as his enteral feeding was re-established and the administration duration of amphotericin B was lengthened to two hours, with no recurrence of arrthymias. 